\begin{tabular}{|c|l|}
\hline Title & Spin Blocker U sing the Interband Rashba Effect in Symmetric Double Quantum Wells \\
\hline Author(s) & Souma, S.; Sawada, A.; Chen, H.; Sekine, Y.; Eto, M.; Koga, T. \\
\hline Citation & $\begin{array}{l}\text { Physical review applied, 4(3), 34010 } \\
\text { https://doi.org/40.1103/PhysRevA pplied.4.034010 }\end{array}$ \\
\hline Issue Date & 2015-09-29 \\
\hline Doc URL & http://hdl.handle.net/2115/60228 \\
\hline Rights & @2015A merican Physical Society \\
\hline Type & article \\
\hline File Information & PhysRevA pplied.4.034010.pdf \\
\hline
\end{tabular}

Instructions for use 


\title{
Spin Blocker Using the Interband Rashba Effect in Symmetric Double Quantum Wells
}

\author{
S. Souma, ${ }^{1}$ A. Sawada, ${ }^{2}$ H. Chen, ${ }^{2}$ Y. Sekine, ${ }^{3}$ M. Eto, ${ }^{4}$ and T. Koga ${ }^{2, *}$ \\ ${ }^{1}$ Department of Electrical and Electronic Engineering, Kobe University, Nada, Kobe 657-8501, Japan \\ ${ }^{2}$ Division of Electronics for Informatics, Graduate School of Information Science and Technology, \\ Hokkaido University, Sapporo, Hokkaido 060-0814, Japan \\ ${ }^{3}$ NTT Basic Research Laboratories, NTT Corporation, Atsugi, Kanagawa 243-0198, Japan \\ ${ }^{4}$ Faculty of Science and Technology, Keio University, Yokohama 223-8522, Japan \\ (Received 23 September 2014; revised manuscript received 12 May 2015; published 29 September 2015)
}

\begin{abstract}
We propose a lateral spin-blockade device that uses the interband Rashba effect in a symmetric double quantum well (QW), where the Rashba effect in the conventional sense vanishes because of its inversion symmetry. The interband Rashba effect manifests itself in the off-diagonal term (represented by the parameter $\eta$ ) in the QW space using the bonding and antibonding basis [Esmerindo Bernardes, John Schliemann, Minchul Lee, J. Carlos Egues, and Daniel Loss, Phys. Rev. Lett. 99, 076603 (2007)]. In such a system, spin selection is possible by tuning the device length, gate electric field and in-plane magnetic field. We particularly show illustrative mechanisms using a one-dimensional model with $\mathbf{k}=\left(k_{F}, 0\right)$, where the selected spin can be blocked completely in the presence of the in-plane magnetic field. While the inclusion of the finite $k_{y}$ and/or the gate electric field deteriorates the spin polarization $P$, finite values remain for $P(P>11 \%)$. Our proposal can also be regarded as an effective way of enhancing a variation of the Rashba-Edelstein effect, the generation of bulk spin polarization by electric current, based on semiconductor band engineering technology.
\end{abstract}

DOI: 10.1103/PhysRevApplied.4.034010

\section{INTRODUCTION}

The generation of spins in nonmagnetic semiconductors is attracting increasing interest not only for applications like the Datta-Das spin-FET [1,2] but also from a basic science viewpoint, such as the confirmation of the RashbaEdelstein effect [3-7], the intrinsic and extrinsic spin-Hall effect [8-11], spin-filtering devices using resonant tunneling diodes [12-14], and spin-dependent phenomena in quantum-point-contact devices [15-18].

In this paper, we utilize the interband Rashba effect in symmetric double quantum wells (DQWs) [14,19-23] to generate a bulk spin polarization nonmagnetically, adopting $\mathrm{In}_{0.53} \mathrm{Ga}_{0.47} \mathrm{As} / \mathrm{In}_{0.52} \mathrm{Al}_{0.48} \mathrm{As}$ DQWs [24] as a specific example. The interband Rashba effect manifests itself in the off-diagonal matrix element in the $2 \times 2$ quantum well (QW) space using the bonding and antibonding basis $[19,20]$, which connects subbands with distinct parities and hence operates without the usual Rashba coupling [25-27]. Because of its novelty, the interband Rashba effect has been providing intriguing playgrounds for physics in various contexts [28,29].

Our DQW-based device has advantages over other nonmagnetic spin-related devices in its reliability (for design and realization) and compatibility with conventional top-down techniques, which are backed up by the well-established semiconductor band engineering technology [30,31]. We specifically aim at enhancing the Rashba-Edelstein effect

\footnotetext{
*koga@ist.hokudai.ac.jp
}

$[3,4]$ to generate a bulk spin polarization in the proposed device. This also circumvents the technological difficulty ( $p$ doping in the middle barrier layer) that one would encounter in the fabrication of the triple-barrier resonant tunneling spin filter [14,32], which shares a common physical basis with the present device.

This paper is organized as follows. We introduce the concept of the proposed device in the next section. We show our theoretical formulation in Sec. III. The results of calculation and discussions are given in Sec. IV. Our conclusions are in Sec. V.

\section{DEVICE DESCRIPTION}

Figures 1(a) and 1(b) illustrate the model structure of the proposed spin device. The active part of the device consists of a (001) InP lattice-matched $\operatorname{In}_{0.53} \mathrm{Ga}_{0.47} \mathrm{As} / \mathrm{In}_{0.52} \mathrm{Al}_{0.48} \mathrm{As}$ DQW [24] with length $L$ in the $x$ direction. Nonmagnetic source and drain electrodes are attached to QW1 after etching away the above layers at both the left and right ends. The $y$ dimension of the device is assumed to be infinite. The QW thickness $d_{\mathrm{QW}}$ is $10 \mathrm{~nm}$ for both QW1 and QW2. The barrier thickness $d_{B}$ between QW1 and QW2 is chosen to be either 2 or $3 \mathrm{~nm}$. Almost equivalently $n$-doped ( $\mathrm{Si}$ ) carrier supplying layers $\left(n^{+} \sim 3 \times 10^{24} \mathrm{~m}^{-3}\right)$ with the thickness $d_{\text {doping }}=$ $6 \mathrm{~nm}$ are placed both above and below the QW layers, separated with nondoped spacers with the thickness $d_{\text {spacer }}=6 \mathrm{~nm}$. Other material-dependent parameters such as the band-gap energy and the conduction band offset are found in Ref. [14]. 


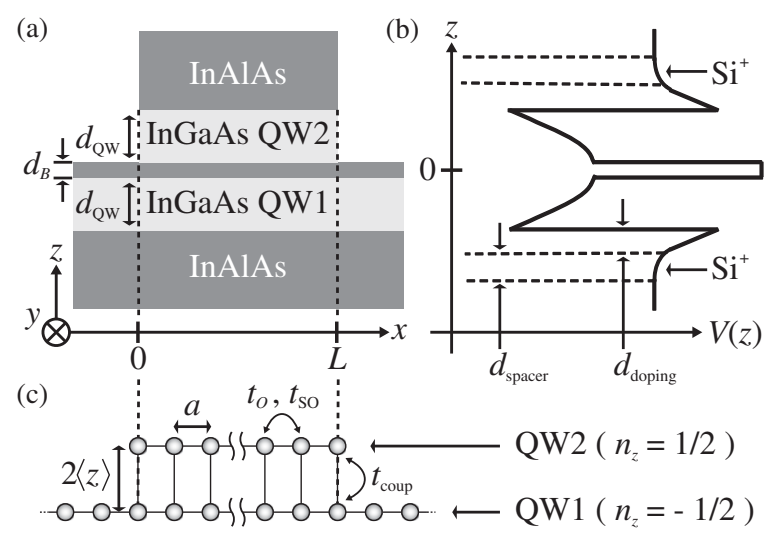

FIG. 1. (a) Model structure of the proposed spin-blocking device made of a DQW. $d_{\mathrm{QW}}=10 \mathrm{~nm}$ and $d_{B}=2$ or $3 \mathrm{~nm}$. (b) Sketch of the potential profile of the symmetric DQW with the ideal doping and gating conditions. $d_{\text {doping }}$ and $d_{\text {spacer }}$ are both $6 \mathrm{~nm}$. The typical dopant density within $d_{\text {doping }}$ is $3 \times 10^{24} \mathrm{~m}^{-3}$. (c) The tight-binding lattice model to simulate the transport property of the electrons along $\hat{x}$ (see Sec. III B for details). The parameter values for $a$ (lattice spacing along $\hat{x}$ ), $t_{o}, t_{\text {so }}, t_{\text {coup }}$ (various interaction parameters), and $2\langle z\rangle$ (lattice spacing along $\hat{z}$ ) are given at the end of Sec. III B.

The fundamental concept behind the proposed device is the spin-dependent energy matching with a common finite $\mathbf{k}$ (in-plane wave vector) in the presence of the opposing relativistic Rashba magnetic fields as in our previous proposal [14]. We would like to increase the internal electric field $\left\langle E_{z}\right\rangle_{1,2}$ within each QW to increase the strength of the Rashba effect through the increase in the sheet carrier density $N_{S}$, while higher subband occupation beyond the first and second ones [(QW1, QW2) or (bonding, antibonding) wave functions along $\hat{z}]$ should be avoided. The Poisson-Schrödinger self-consistent simulation predicts that $N_{S}=3.6 \times 10^{16} \mathrm{~m}^{-2}\left(E_{F}=91.7 \mathrm{meV}\right.$ for the Fermi energy) gives a reasonably large value for the interband Rashba coefficient $\eta=3.14 \times 10^{-12} \mathrm{eV} \mathrm{m}$ for the two subband system. If the two wells are completely independent, the $\eta$ value is virtually the same as that of the regular Rashba coefficient $\alpha$ in each constituent QW apart from its sign. We find that the bottom of the third subband is located $40 \mathrm{meV}$ above $E_{F}$, so that the two-band approximation suffices for realistic predictions of the spindependent transport properties. We also recently found that the Dresselhaus effect is negligible relative to the Rashba effect in this system, hence no inclusion of the Dresselhaus effect in the present work [27].

\section{A. Effective Hamiltonian for the DQW}

Using the bonding and antibonding basis in the $2 \times 2$ QW space [Fig. 2(a)], the effective Hamiltonian $H$ for the DQW in the presence of a magnetic field $\mathbf{B} \| \hat{y}$ can be decomposed into $H_{0}+H_{B_{\|}}+H_{R}+H_{Z}$, where

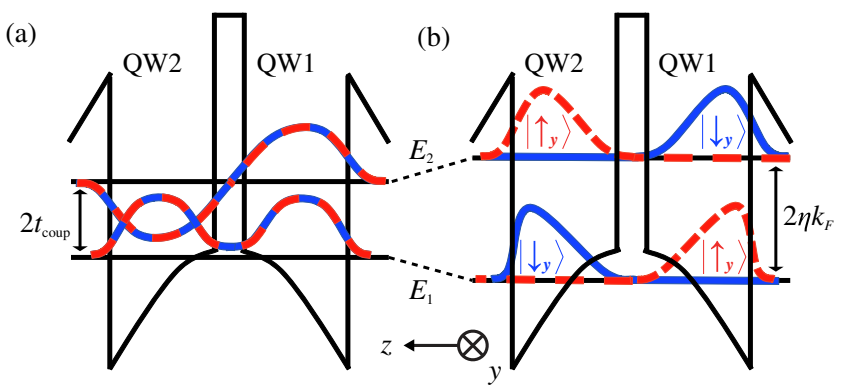

FIG. 2. Sketches of electron eigenfunctions for a symmetric DQW along $\hat{z}$ with $\mathbf{B}=0$. (a) The case without the interband Rashba effect or with $\mathbf{k}=(0,0)$ for the in-plane wave vector, where the eigenfunctions become spin-independent and bonding and antibonding like [blue-red curves]. The interaction parameter $t_{\text {coup }}$ is defined as the half of the difference in the eigenenergies $\left(E_{2}-E_{1}\right)$. (b) The case with a finite interband Rashba effect and $\mathbf{k}=\left(k_{F}, 0\right)$. Assuming $2 t_{\text {coup }} \ll 2 \eta k_{F}$ (interband Rashba splitting energy), $E_{2}-E_{1} \approx 2 \eta k_{F}$. While the spin degeneracy $[E(\mathbf{k}, \uparrow)=E(\mathbf{k}, \downarrow)]$ is preserved due to the inversion symmetry, the eigenfunctions along $\hat{z}$ for the same eigenenergy $\left(E_{1}\right.$ or $\left.E_{2}\right)$ are spin dependent (red broken and blue solid curves for spin up and down, respectively).

$$
\begin{aligned}
H_{0} & =\frac{\hbar^{2}}{2 m_{\|}^{*}}\left(\hat{k}_{x}^{2}+\hat{k}_{y}^{2}\right)\left(\begin{array}{ll}
1 & 0 \\
0 & 1
\end{array}\right)_{\mathrm{QW}}+t_{\mathrm{coup}}\left(\begin{array}{cc}
-1 & 0 \\
0 & 1
\end{array}\right)_{\mathrm{QW}}, \\
H_{B_{\|}} & =-\frac{e B \hbar \hat{k}_{x}\langle z\rangle}{m_{\|}^{*}}\left(\begin{array}{ll}
0 & 1 \\
1 & 0
\end{array}\right)_{\mathrm{QW}}+\frac{e^{2} B^{2}\left\langle z^{2}\right\rangle}{2 m_{\|}^{*}}\left(\begin{array}{ll}
1 & 0 \\
0 & 1
\end{array}\right)_{\mathrm{QW}}, \\
H_{R} & =\eta\left(\hat{k}_{y} \sigma_{x}-\hat{k}_{x} \sigma_{y}\right)\left(\begin{array}{ll}
0 & 1 \\
1 & 0
\end{array}\right)_{\mathrm{QW}},
\end{aligned}
$$

and

$$
H_{Z}=\frac{1}{2} g^{*} \mu_{B} B \sigma_{y}\left(\begin{array}{ll}
1 & 0 \\
0 & 1
\end{array}\right)_{\mathrm{QW}} .
$$

The in-plane field $\mathbf{B}$, incorporated by setting $\mathbf{p} \rightarrow$ $\mathbf{p}+e \mathbf{A}$ with $\quad \mathbf{A}=(B z, 0,0) \quad[\mathbf{B}=\nabla \times \mathbf{A}=(0, B, 0)]$, which results in $H_{B_{\|}}$, provides the essential mechanism for spin blocking. $H_{0}, H_{R}$, and $H_{Z}$ are the unperturbed, interband Rashba, and Zeeman Hamiltonians, respectively. $\mu_{B}=e \hbar / 2 m_{e}$ is the Bohr magneton ( $m_{e}$ being the free electron mass), $g^{*}$ is the effective $g$-factor value, and $m_{\|}^{*}$ is the in-plane effective mass. $\hat{k}_{x}$ and $\hat{k}_{y}$ are $\partial / i \partial x$ and $\partial / i \partial y$, respectively. $e, \hbar, \sigma_{x}$, and $\sigma_{y}$ are the elementary charge, Planck's constant divided by $2 \pi$, and the Pauli spin matrices, respectively. We set the origin of $z$ at the middle of the barrier layer to define $\langle z\rangle \equiv\left\langle\Phi_{\mathrm{QW}_{2}}|z| \Phi_{\mathrm{QW}_{2}}\right\rangle=-\left\langle\Phi_{\mathrm{QW}_{1} \mid}|z| \Phi_{\mathrm{QW} 1}\right\rangle>0$. For our $\mathrm{In}_{0.53} \mathrm{Ga}_{0.47} \mathrm{As} / \mathrm{In}_{0.52} \mathrm{Al}_{0.48} \mathrm{As} \mathrm{DQW}$, we safely ignore $H_{Z}$ and $e^{2} B^{2}\left\langle z^{2}\right\rangle / 2 m_{\|}^{*}$ in $H_{B_{\|}}\left(\ll H_{R}, H_{0}\right)$. We also neglected $e B\langle z\rangle / \hbar$ in $H_{R}$ relative to $k_{x}$. These are valid approximations in the range of $B$ and $k_{x}$ of our interest [33]. 
The interwell coupling $t_{\text {coup }}$ in Eq. (1) is defined by $\left(E_{2}-E_{1}\right) / 2$, letting $E_{1}$ and $E_{2}$ be the eigenvalues of $-(d / d z)\left[\hbar^{2} / 2 m_{z}^{*}(z)\right](d / d z)+V(z)$ [see Fig. 2(a)], where $m_{z}^{*}(z)$ incorporates different effective masses between the well and barrier layers. $V(z)$ describes the DQW confinement potential including the Hartree term, which possesses the inversion symmetry $V(z)=V(-z)$ [Fig. 1(b)]. We use the function $a_{\mathrm{so}} E_{z}(z)$ [27] for the estimation of the interband Rashba parameter $\eta$, where $a_{\mathrm{so}}$ and $E_{z}(z)$ are the intrinsic constant for the Rashba effect and the electric field perpendicular to the interface as a function of $z$, respectively. We note that more rigorous treatments exist for the interband Rashba effect in DQWs [19,20]. Our treatment here incorporates the first-order correction about the interfacial band offsets [34,35]; thus, the $a_{\mathrm{so}}$ value is about $40 \%$ larger than its bulk counterpart $\mathrm{r}_{41}^{6 c 6 c}$ (see Refs. [26,34]). $E_{z}(z)$ is given by the Gauss law $E_{z}(z)=$ $\varepsilon^{-1}(z) \int_{0}^{z} \rho\left(z^{\prime}\right) d z^{\prime}$, where $\varepsilon(z)$ and $\rho(z)$ are the dielectric constant and net charge density as a function of $z$, respectively. $E_{z}(z)$ is antisymmetric about $z=0$; i.e., $E_{z}(z)=-E_{z}(-z)$. The $\eta$ value for symmetric DQWs is given as $\left\langle b\left|a_{\mathrm{so}} E_{z}(z)\right| a b\right\rangle$, where $|b\rangle$ and $|a b\rangle$ are the bonding and antibonding wave functions along $\hat{z}$, respectively.

\section{B. Eigenfunctions of $\boldsymbol{H}$ without a magnetic field}

We first set $B=0$, where $H$ can be diagonalized in spin space using the spin basis

$$
\left|\sigma_{ \pm}\right\rangle=\frac{1}{\sqrt{2} k}\left(\begin{array}{c}
k_{y}+i k_{x} \\
\mp k
\end{array}\right) .
$$

Then, we obtain

$$
\left[H-\frac{\hbar^{2} k^{2}}{2 m_{\|}^{*}}\right]=\left(\begin{array}{cc}
-t_{\text {coup }} & \mp \eta k \\
\mp \eta k & t_{\text {coup }}
\end{array}\right)_{\mathrm{QW}}
$$

in the QW space using the bonding or antibonding basis, which can be solved for each spin $\left|\sigma_{ \pm}\right\rangle$separately (doublesign corresponds) [see Fig. 2(b)]. $\hat{k}_{x}$ and $\hat{k}_{y}$ are replaced with the wave numbers $k_{x}$ and $k_{y}$, because they commute with $H$. The inversion symmetry in the DQW, i.e., $V(z)=V(-z)$, ensures the spin degeneracy $E\left(\mathbf{k}, \sigma_{+}\right)=$ $E\left(\mathbf{k}, \sigma_{-}\right)$.

Taking advantage of the circular symmetry, we consider only the case $\mathbf{k}=\left(k_{F}, 0\right)$ without the loss of generality, where the spin basis $\left|\sigma_{+}\right\rangle$and $\left|\sigma_{-}\right\rangle$becomes $\left|\uparrow_{y}\right\rangle$ ("up") and $\left|\downarrow_{y}\right\rangle$ ("down") in $\hat{y}$, respectively. We find two eigenenergies $E_{1}=\left(\hbar^{2} k_{F}^{2} / 2 m_{\|}^{*}\right)-\sqrt{t_{\text {coup }}^{2}+\eta^{2} k_{F}^{2}}$ and $E_{2}=\left(\hbar^{2} k_{F}^{2} / 2 m_{\|}^{*}\right)+$ $\sqrt{t_{\text {coup }}^{2}+\eta^{2} k_{F}^{2}}$ for either spin, hence the spin degeneracy. The orbital eigenfunctions along $\hat{z}$ for spin up $\left|\Phi_{1 \uparrow}(z)\right\rangle$ and $\left|\Phi_{2 \uparrow}(z)\right\rangle$, associated with $E_{1}$ and $E_{2}$, respectively, are

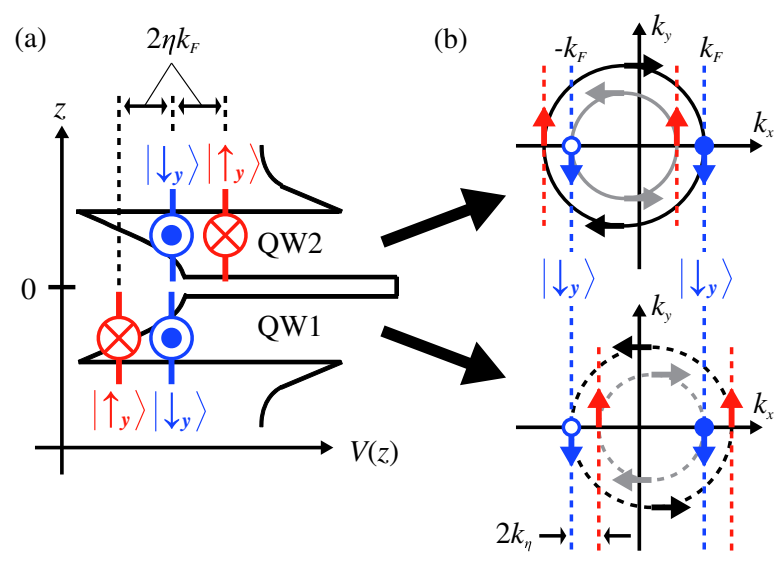

FIG. 3. (a) Spin-dependent eigenenergies of the DQW at $\mathbf{k}=$ $\left(k_{F}, 0\right)$ and $\mathbf{B}=\left(0, B_{a}, 0\right)$, assuming $t_{\text {coup }} \approx 0$ (see Fig. 2 for $\left.t_{\text {coup }}\right)$. The point $\mathbf{k}=\left(k_{F}, 0\right)$ in the canonical $\mathbf{k}$ space is indicated by the blue filled circles (dots) in (b). (b) Spin-split Fermi circles of the DQW with $t_{\text {coup }} \approx 0$ and $\mathbf{B}=\left(0, B_{a}, 0\right)$, where indicated by the arrows on the circles are the spin orientations at each $\mathbf{k}$ point. The open and filled blue circles (dots) indicate $\mathbf{k}=\left( \pm k_{F}, 0\right)$ points, where only the down-spin electrons form bonding and antibonding eigenstates between QW1 and QW2.

mirror images of the spin-down counterparts, i.e., $\left|\Phi_{1 \uparrow}(z)\right\rangle=\left|\Phi_{1 \downarrow}(-z)\right\rangle \quad$ and $\quad\left|\Phi_{2 \uparrow}(z)\right\rangle=\left|\Phi_{2 \downarrow}(-z)\right\rangle \quad$ [see Fig. 2(b)]. We define $\left|\Phi_{\mathrm{QW1}}\right\rangle \equiv(1 / \sqrt{2})(|b\rangle+|a b\rangle)$ and $\left|\Phi_{\mathrm{QW} 2}\right\rangle \equiv(1 / \sqrt{2})(|b\rangle-|a b\rangle)$ so that $\left|\Phi_{1 \uparrow}(z)\right\rangle,\left|\Phi_{2 \downarrow}(z)\right\rangle \rightarrow$ $\left|\Phi_{\mathrm{QW} 1}\right\rangle$ and $\left|\Phi_{1 \downarrow}(z)\right\rangle,\left|\Phi_{2 \uparrow}(z)\right\rangle \rightarrow\left|\Phi_{\mathrm{QW} 2}\right\rangle$ for $t_{\text {coup }} \rightarrow 0$. Assuming $t_{\text {coup }} / \eta k_{F} \ll 1$, we approximate $\left|\Phi_{1 \uparrow, \downarrow}\right\rangle$ and $\left|\Phi_{2 \uparrow, \downarrow}\right\rangle$ by $\left|\Phi_{\mathrm{QW} 1,2}\right\rangle$ hereafter unless otherwise mentioned. We define the Rashba wave number $k_{\eta} \equiv m_{\|}^{*} \eta / \hbar^{2}$. The difference in radius between the inner and outer circles in Fig. 3(b) is $2 k_{\eta}$.

\section{Mechanism of spin blocking by the in-plane magnetic field}

Equation (1) can be interpreted in such a way that an inplane magnetic field $(\mathbf{B} \| \hat{y})$ shifts the Fermi circles of QW1 and QW2 in the canonical $k_{x}$ direction oppositely by the magnitude $e B\langle z\rangle / \hbar$ as shown in Fig. 3(b) [36]. The spinselective resonant states between QW1 and QW2 are formed at $\mathbf{k}=\left( \pm k_{F}, 0\right)$ if the shift of the Fermi circle by $\mathbf{B}$ becomes equal to $k_{\eta}$, where $k_{\eta} \equiv m_{\|}^{*} \eta / \hbar^{2}$ and $\langle z\rangle \equiv$ $-\langle b|z| a b\rangle>0$ using the bonding and antibonding basis [see the filled and open blue circles (dots) in Fig. 3(b)]. We call $B_{a} \equiv \hbar k_{\eta} / e\langle z\rangle$ the anticrossing magnetic field, at which the energy levels for the selected spin anticross.

Let us consider the propagation of an electron with $\mathbf{k}=\left(k_{x}, 0\right)$ at the Fermi energy in the presence of $\mathbf{B}=\left(0, B_{a}, 0\right)$, injected to QW1 at $x=0$ [see Fig. 4(a) for the down-spin propagation]. The spin-dependent dispersion relations of a DQW with this condition are sketched in Fig. 4(b), where the wave number values of $k_{x}$ at the 
(a)

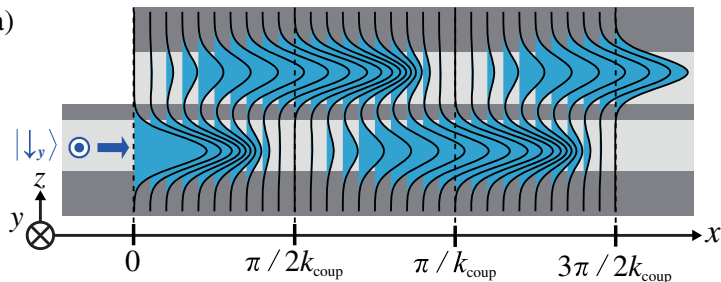

(b)

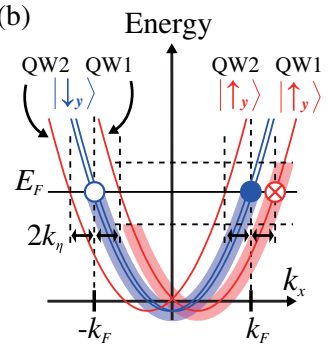

(c)

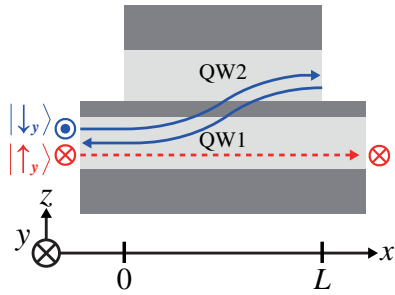

(d) Energy

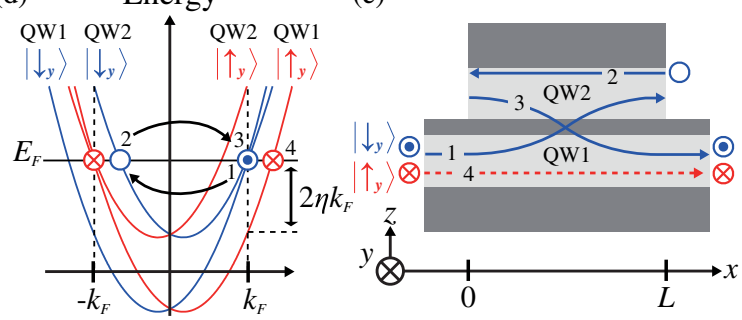

FIG. 4. (a) Sketch of $\left|\Psi_{\text {sup }}\right|^{2}$ [see Eq. (2)] as a function of $x$. (b) Energy dispersion relation of the proposed DQW when the in-plane magnetic field $\mathbf{B}=\left(0, B_{a}, 0\right)$ is applied. The spin-down branches (blue curves) are nearly degenerate for all $k_{x}$ 's. The hatching on the dispersion curves expresses the imbalance of the electron occupation by the steady current $\mathbf{I} \| \hat{x}$ for the device shown in (c). (c) Spin-dependent trajectories of an electron injected in QW1 from the left in the presence of $\mathbf{B}=\left(0, B_{a}, 0\right)$. (d) The energy dispersion relation realized by gating, where the interband Rashba splitting energy $2 \eta k_{F}$ (exaggerated vertically for clarity) is compensated by different site potential energies $\Delta V(z)$ at $\mathrm{QW} 1$ and QW2, i.e., $\Delta V(\langle z\rangle)=-\Delta V(-\langle z\rangle)=\eta k_{F}$. Thus, the branches associated with QW2 and QW1 (labeled in the figure) are moved upward and downward, respectively, by $\eta k_{F}$. (e) Spin-dependent trajectories of an electron injected in QW1 from the left with the band structures as described in (d). The motions of spin-up and -down electrons are indicated by the number(s) "4" and "1, 2, 3," respectively, in (d) and (e).

Fermi energy $\left[E_{F}=\left(\hbar^{2} k_{F}^{2} / 2 m^{*}\right)\right]$ for spin-up electrons are $-k_{F}-2 k_{\eta},-k_{F}+2 k_{\eta}, k_{F}-2 k_{\eta}$, and $k_{F}+2 k_{\eta}$ (nondegenerate), while those for spin down are $-k_{F} \mp k_{\text {coup }}$ and $+k_{F} \pm k_{\text {coup }}$ (nearly doubly degenerate at each $k_{x}= \pm k_{F}$ ) for $t_{\text {coup }} \rightarrow 0$, where $k_{\text {coup }} \equiv t_{\text {coup }}\left[d E\left(k_{x}\right) / d k_{x}\right]^{-1}$. The states with $k_{x}= \pm k_{F}-2 k_{\eta}$ (up spin in $\hat{y}$ ) have a wave function along $\hat{z}$ as $\left|\Phi_{\mathrm{QW} 2}\right\rangle \equiv(1 / \sqrt{2})(|b\rangle-|a b\rangle)$, while those with $\pm k_{F}+2 k_{\eta}$ (also up spin in $\hat{y}$ ) as $\left|\Phi_{\mathrm{QW} 1}\right\rangle \equiv(1 / \sqrt{2})(|b\rangle+$ $|a b\rangle)$. The down-spin electrons with $k_{x}=-k_{F} \mp k_{\text {coup }}$ or $k_{x}=k_{F} \pm k_{\text {coup }}$ (down spin in $\hat{y}$ ), on the other hand, form bonding and antibonding eigenstates between QW1 and QW2 when $t_{\text {coup }} \approx 0$ (small, but finite). The equally weighted superposition of these bonding and antibonding wave functions including the plane wave parts in $\hat{x}$ $\left(k_{x}>0\right)$, which are $e^{i\left(k_{F}+k_{\text {coup }}\right) x}$ and $e^{i\left(k_{F}-k_{\text {coup }}\right) x}$, respectively, reads

$$
\Psi_{\text {sup }}=\frac{1}{\sqrt{2}}\left\{e^{i\left(k_{F}+k_{\text {coup }}\right) x}|b\rangle+e^{i\left(k_{F}-k_{\text {coup }}\right) x}|a b\rangle\right\} .
$$

$\Psi_{\text {sup }}$ can be simplified to $e^{i k_{F} x}\left\{\cos \left(k_{\text {coup }} x\right)\left|\Phi_{\mathrm{QW} 1}\right\rangle+\right.$ $\left.i \sin \left(k_{\text {coup }} x\right)\left|\Phi_{\mathrm{QW} 2}\right\rangle\right\}$ after some algebra. We note $\Psi_{\text {sup }}=$ $\left|\Phi_{\mathrm{QW} 1}\right\rangle$ at $x=0$. Thus, $\Psi_{\text {sup }}$ describes the propagation of down-spin electrons injected to QW1 at $x=0$ as in Fig. 4(a) [37].

We find that such a precessional electron [solid blue circle in Fig. 4(b) in canonical $k$ space] is backscattered at $x=L$, where we have the reflection wall in QW2 as in Fig. 4(c), if the condition $L=L_{n} \equiv\left(n-\frac{1}{2}\right) \pi / k_{\text {coup }}$ is satisfied [see Fig. 4(a)]. The backscattered electron, where spin is assumed to be preserved by reflection, now propagates backward in the DQW [blue open circle in Fig. 4(b)], precessing between QW1 and QW2 again, keeping its spin state unaltered. The corresponding motion of the electron in real space is sketched by the blue curves with an arrow in Fig. 4(c). Electrons with up spin, on the other hand, injected from the left lead in QW1, are transmitted straight to the right lead [red circled times in Figs. 4(b) and 4(c)]. Thus, the population imbalance between the positive and negative $k_{x}$ 's is formed only for the QW1 branch of the up-spin electrons as in Fig. 4(b). This provides explicitly the mechanism of spin blocking and results in current-induced bulk spin polarization.

We note that the spin-blocking effect described here cannot be realized electrically (i.e., by a gate) in this simple one-dimensional limit [38]. Here, we consider the additional site potential energy $\Delta V(z)$, by the modified gate electric field $\Delta E_{z}\left[\Delta V(z)=\Delta E_{z} z\right]$ in the DQW, that exactly compensates the interband Rashba spin splitting energy $2 \eta k_{F}$, i.e., $\quad \Delta V(\langle z\rangle)=-\Delta V(-\langle z\rangle)=\eta k_{F} \quad$ [see Fig. 4(d)]. We note that $\left|\Delta E_{z}\right| \ll\left\langle E_{z}\right\rangle=\left\langle b\left|E_{z}(z)\right| a b\right\rangle$ in our particular model, so that the $\eta$ value is assumed to be unchanged by $\Delta E_{z}$. In this case, the precessional down-spin electrons like $\Psi_{\text {sup }}$ [Eq. (2)] can be formed at $\mathbf{k}=\left(k_{F}, 0\right)$ as in the case of the application of $\mathbf{B}=\left(0, B_{a}, 0\right)$. However, these electrons cannot be backscattered to the empty precessional states at $\mathbf{k}=\left(-k_{F}, 0\right)$ because the spin state there (red circled times) is orthogonal to the spin state of the reflected electrons (blue empty circle) as in Fig. 4(d). The detailed motions of the corresponding spin-up and -down electrons in real space are also shown in Fig. 4(e).

\section{THEORETICAL FORMULATION}

Here we describe the theoretical models to calculate the spin-dependent transport of the proposed device. A 
tight-binding (TB) model may be advantageous when treating the multiplicity of the transport channel (with finite $k_{y}$ 's), or even arbitrary-shaped nanostructures. A multiple-reflection (MR) model is more favorable for acquiring physical intuitions. TB and MR produce identical results in the one-dimensional limit with $k_{y}=0$. The extension of these models to full two-dimensional versions is straightforward but may require some approximations in performing actual calculations.

\section{A. Spin-dependent conductance}

We define the spin-dependent conductance $G_{\uparrow, \downarrow}$ associated with the right lead by $I_{\uparrow, \downarrow}=G_{\uparrow, \downarrow} V_{\text {sd }}$, where $I_{\uparrow, \downarrow}$ is the spin-dependent current in the right lead and $V_{\text {sd }}$ is the source-drain voltage. Using the Landauer-Büttiker formalism [39], $G_{\uparrow, \downarrow}$ can be written as

$G_{\sigma}=\frac{e^{2}}{h} \sum_{k_{y}} \sum_{\sigma^{\prime}=\uparrow, \downarrow} \int d E T_{\sigma \sigma^{\prime}, k_{y}}(E)\left\{-\frac{d f\left(E-E_{F}\right)}{d E}\right\}$,

where $T_{\sigma \sigma^{\prime}, k_{y}}(E)$ is the transmission probability of an electron from the spin state $\sigma^{\prime}\left(\sigma^{\prime}=\uparrow, \downarrow\right)$ in the left lead to the spin state $\sigma(\sigma=\uparrow, \downarrow)$ in the right lead, assuming the conservation of $k_{y}$ during the transmission. The spin basis here can be chosen to be one of any orthogonal sets at one's convenience. The thermal smearing of the Fermi surface is included in Eq. (3) through the Fermi distribution function $f\left(E-E_{F}\right)$.

The spin polarization of the electric current in the right lead is given by

$$
P=\frac{I_{\uparrow}-I_{\downarrow}}{I_{\uparrow}+I_{\downarrow}}=\frac{G_{\uparrow}-G_{\downarrow}}{G_{\uparrow}+G_{\downarrow}} .
$$

The actual calculation of $T_{\sigma \sigma^{\prime}, k_{y}}$ is performed by the recursive Green function technique using the TB Hamiltonian [40] (Sec. III B) or by the MR model (Sec. III C).

\section{B. Tight-binding model}

Keeping in mind that the proposed spin-filtering device has translational symmetry along the $y$ direction, the TB Hamiltonian [40] including $k_{y}$ as a constant [also see Fig. 1(c)] is given as

$$
\hat{H}_{k_{y}}=\sum_{\mathbf{n}, \sigma, \sigma^{\prime}} \epsilon_{\mathbf{n} k_{y}}^{\sigma \sigma^{\prime}} \hat{c}_{\mathbf{n} \sigma}^{\dagger} \hat{c}_{\mathbf{n} \sigma^{\prime}}+\sum_{\mathbf{n}, \mathbf{n}^{\prime}, \sigma, \sigma^{\prime}} \hat{c}_{\mathbf{n} \sigma}^{\dagger} t_{\mathbf{n}^{\prime}}^{\sigma \sigma^{\prime}} \hat{c}_{\mathbf{n}^{\prime} \sigma^{\prime}}
$$

where

$$
\epsilon_{\mathbf{n} k_{y}}^{\sigma \sigma^{\prime}}=\left[\frac{\hbar^{2} k_{y}^{2}}{2 m_{\|}^{*}}+2 t_{o}\right] \delta_{\sigma \sigma^{\prime}}+2 a t_{\mathrm{so}}\left(n_{z}\right) k_{y}\left[\sigma_{x}\right]_{\sigma \sigma^{\prime}}
$$

and

$$
t_{\mathbf{n n}^{\prime}}^{\sigma \sigma^{\prime}}= \begin{cases}\left\{-t_{o} \delta_{\sigma \sigma^{\prime}} \mp i t_{\mathrm{so}}\left(n_{z}\right)\left[\sigma_{y}\right]_{\sigma \sigma^{\prime}}\right\} & \\ \times \exp \left[\mp i \phi\left(n_{z}\right)\right] & \left(\mathbf{n}=\mathbf{n}^{\prime} \pm \mathbf{e}_{x}\right), \\ -t_{\operatorname{coup}} \delta_{\sigma \sigma^{\prime}} & \left(\mathbf{n}=\mathbf{n}^{\prime} \pm \mathbf{e}_{z}\right), \\ 0 & (\text { otherwise }) .\end{cases}
$$

Here, $\hat{c}_{\mathbf{n} \sigma}\left(\hat{c}_{\mathbf{n} \sigma}^{\dagger}\right)$ is the annihilation (creation) operator of an electron at site $\mathbf{n}=\left(n_{x}, n_{z}\right)$ with spin $\sigma(=\uparrow, \downarrow)$, where $n_{x}$ is an integer, $n_{z}= \pm 1 / 2$, and the spin basis in the present work is chosen in the $y$ direction. $a, t_{o} \equiv \hbar^{2} / 2 m_{\|}^{*} a^{2}$, and $t_{\mathrm{so}}\left(n_{z}\right)=$ $n_{z} \eta / a$ are the lattice spacing along $\hat{x}$, the orbital hopping parameter, and the hopping parameter associated with the Rashba effect, respectively. The tunneling between QW1 and QW2 is characterized by $t_{\text {coup }}$ (Sec. II B). The magnetic field is incorporated as a Peierls phase factor $\phi\left(n_{z}\right)=n_{z} \pi \Phi / \Phi_{0}$, where $\Phi_{0}=h / e$ and $\Phi=2 B a\langle z\rangle$ with $\mathbf{B}=(0, B, 0)$. We do not include the Rashba effect and $\mathbf{B}$ in the leads $(x<0$ and $x>L$ ). The actual TB calculation is performed for the total sheet carrier density $N_{S}=3.6 \times 10^{16} \mathrm{~m}^{-2}$ and $a=1 \mathrm{~nm}$. The parameter values in this case are $t_{o}=0.81 \mathrm{eV}$, $t_{\mathrm{so}}=1.57 \mathrm{meV}, t_{\text {coup }}=0.44 \mathrm{meV}$, and $\langle z\rangle=7.21 \mathrm{~nm}$ for $d_{B}=3 \mathrm{~nm} \mathrm{[41].}$

\section{Multiple-reflection model}

Let $t_{n n^{\prime}}\left(n, n^{\prime}=1\right.$ or 2$)$ be the nominal quantummechanical transmission amplitude of an electron from $x=0$ in $\mathrm{QW} n^{\prime}$ to $x=L$ in $\mathrm{QW} n$. Similarly, let $r_{n n^{\prime}}$ be the nominal transmission amplitude of an electron from $x=L$ in $\mathrm{QW} n^{\prime}$ to $x=0$ in $\mathrm{QW} n$. The MR model states that the overall transmission amplitude from QW1 at $x=0$ to QW1 at $x=L$ is given by

$$
\begin{aligned}
t_{\mathrm{tot}} & =t_{11}+t_{12} r_{22} t_{21}+t_{12} r_{22} t_{22} r_{22} t_{21}+\cdots \\
& =t_{11}+t_{12} r_{22}\left\{1+\left(t_{22} r_{22}\right)+\left(t_{22} r_{22}\right)^{2}+\cdots\right\} t_{21} \\
& =t_{11}+\frac{t_{12} r_{22} t_{21}}{1-t_{22} r_{22}} .
\end{aligned}
$$

The overall transmission probability $T_{\text {tot }}$ is given by $\left|t_{\text {tot }}\right|^{2}$. The values of $t_{n n^{\prime}}$ and $r_{n n^{\prime}}$ are evaluated for each spin separately as below.

Choosing the spin basis in $\hat{y}$ and assuming $\mathbf{k}=\left(k_{x}, 0\right)$, we can write the eigenfunctions of the DQW with $E=E_{F}$ and $\mathbf{B}=\left(0, B_{a}, 0\right)$ as $e^{ \pm i\left(k_{F}+\Delta k\right) x}\left|\Phi_{b}\right\rangle$ and $e^{ \pm i\left(k_{F}-\Delta k\right) x}\left|\Phi_{a}\right\rangle$ for either spin, where $\Delta k\left(=k_{\text {coup }}\right.$ or $\left.2 k_{\eta}^{\prime}\right)$ and $\left|\Phi_{b, a}\right\rangle$ are the positive eigenvalue and the eigenfunctions of

$$
\left(\begin{array}{cc}
-k_{\text {coup }} & 0 \\
0 & k_{\text {coup }}
\end{array}\right)_{\mathrm{QW}}
$$

(for spin down) or those of 


$$
\left(\begin{array}{cc}
-k_{\text {coup }} & -2 k_{\eta} \\
-2 k_{\eta} & k_{\text {coup }}
\end{array}\right)_{\mathrm{QW}}
$$

(for spin up), respectively, in the QW space (bonding and antibonding basis). We find that $k_{\eta}^{\prime}=\sqrt{k_{\eta}^{2}+\frac{1}{4} k_{\text {coup }}^{2}}$ and that

$$
\left|\Phi_{b}\right\rangle=\sqrt{1-\delta^{2}}\left|\Phi_{\mathrm{QW} 1}\right\rangle+\delta\left|\Phi_{\mathrm{QW} 2}\right\rangle
$$

and

$$
\left|\Phi_{a}\right\rangle=\delta\left|\Phi_{\mathrm{QW} 1}\right\rangle-\sqrt{1-\delta^{2}}\left|\Phi_{\mathrm{QW} 2}\right\rangle
$$

with $0<\delta<1$ using the QW1 and QW2 basis. We further note that $\delta=1 / \sqrt{2}$ for spin down; therefore, $\left|\Phi_{b}\right\rangle=|b\rangle$ and $\left|\Phi_{a}\right\rangle=|a b\rangle$. We solve Eqs. (9) and (10) for $\left|\Phi_{\mathrm{QW} 1}\right\rangle$ and $\left|\Phi_{\mathrm{QW} 2}\right\rangle$, and let these wave functions propagate from $x=0$ to $x=L$ :

$$
\begin{aligned}
& \left|\Phi_{\mathrm{QW} 1}\right\rangle \quad(\text { at } x=0) \\
& \rightarrow e^{i k_{F} L}\left\{e^{i \Delta k L} \sqrt{1-\delta^{2}}\left|\Phi_{b}\right\rangle+e^{-i \Delta k L} \delta\left|\Phi_{a}\right\rangle\right\} \\
& =e^{i k_{F} L}\left[\left\{e^{i \Delta k L}-2 i \delta^{2} \sin (\Delta k L)\right\}\left|\Phi_{\mathrm{QW} 1}\right\rangle\right. \\
& \left.\quad+2 i \delta \sqrt{1-\delta^{2}} \sin (\Delta k L)\left|\Phi_{\mathrm{QW} 2}\right\rangle\right] \quad(\text { at } x=L), \\
& \left|\Phi_{\mathrm{QW} 2}\right\rangle \quad(\text { at } x=0) \\
& \rightarrow e^{i k_{F} L}\left\{e^{i \Delta k L} \delta\left|\Phi_{b}\right\rangle+e^{-i \Delta k L} \sqrt{1-\delta^{2}}\left|\Phi_{a}\right\rangle\right\} \\
& =e^{i k_{F} L}\left[2 i \delta \sqrt{1-\delta^{2}} \sin (\Delta k L)\left|\Phi_{\mathrm{QW} 1}\right\rangle\right. \\
& \left.\quad+\left\{e^{-i \Delta k L}+2 i \delta^{2} \sin (\Delta k L)\right\}\left|\Phi_{\mathrm{QW} 2}\right\rangle\right] \quad(\text { at } x=L) .
\end{aligned}
$$

From these we obtain

$$
\begin{aligned}
t_{11} & =e^{2 i k_{F} L} t_{22}^{*} \\
& =e^{i k_{F} L}\left\{e^{i \Delta k L}-2 i \delta^{2} \sin (\Delta k L)\right\}, \\
t_{12} & =t_{21} \\
& =e^{i k_{F} L}\left\{2 i \delta \sqrt{1-\delta^{2}} \sin (\Delta k L)\right\} .
\end{aligned}
$$

Now we let $\left|\Phi_{\mathrm{QW} 2}\right\rangle$ propagate from $x=L$ to $x=0$ to obtain $r_{22}$. After a simple symmetry consideration as in Fig. 5, we notice that it is equal to $t_{11}$. Substituting Eqs. (12) and (13) into Eq. (8), we obtain

$$
t_{\mathrm{tot}}=\frac{e^{i k_{F} L}\left(1-e^{2 i k_{F} L}\right)\left[e^{i \Delta k L}-i \delta^{2} \sin \left(k_{F} L\right)\right]}{1-e^{2 i k_{F} L} T_{11}},
$$

where

$$
T_{11}=\left|t_{11}\right|^{2}=1-4 \delta^{2}\left(1-\delta^{2}\right) \sin ^{2}(\Delta k L) .
$$
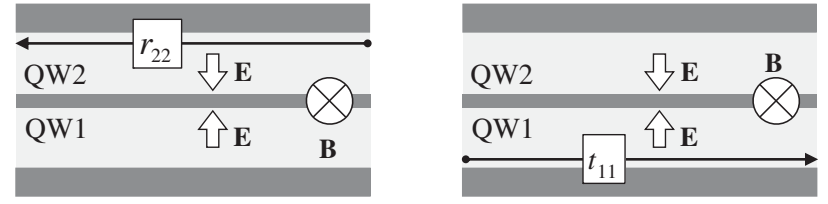

FIG. 5. Illustration to show $r_{22}=t_{11}$ from symmetry consideration, where $\mathbf{E}$ and $\mathbf{B}$ are the built-in electric field derived from the confinement potential $V(z)$ and the external magnetic field, respectively. $t_{11}$ and $r_{22}$ are the quantum-mechanical transmission or reflection amplitudes in QW1 and QW2, respectively.

The overall transmission probability per spin is

$$
T_{\mathrm{tot}}=\left|t_{\mathrm{tot}}\right|^{2}=\frac{2\left[1-\cos \left(2 k_{F} L\right)\right] T_{11}}{1-2 T_{11} \cos \left(2 k_{F} L\right)+T_{11}^{2}} .
$$

We confirmed that this reproduces the TB results as shown in the next section. We note that the spin dependence of $T_{\text {tot }}$ appears only through those of $\delta$ and $\Delta k$ in $T_{11}$. The thermal average of $T_{\text {tot }}$ is also straightforward.

\section{RESULTS OF THE CALCULATION}

First, we restrict our discussion to the case $k_{y}=0$ and skip the summation over $k_{y}$ in Eq. (3). In this case, $T_{\uparrow \downarrow, 0}(E)=$ $T_{\downarrow \uparrow, 0}(E)=0$ if we choose the spin basis in $\hat{y}$, which largely simplifies the problem. Plotted in Fig. 6 are the calculated $G_{\uparrow, \downarrow}$ as a function of $L$ at $B=B_{a}$ for $d_{B}=2$ and $3 \mathrm{~nm}$ $(T=5 \mathrm{~K})$. The change of $G_{\downarrow}$ as a function of $L$ is more pronounced than that of $G_{\uparrow}$, where $G_{\downarrow}$ even becomes zero at specific device lengths $L_{n}^{*}, n$ being a positive integer, while $G_{\uparrow}$ varies only weakly with $L$. These qualitative behaviors agree with the argument in Sec. II C. We find that $L_{1}^{*}=$ $648 \mathrm{~nm}$ and $L_{2}^{*}=1946 \mathrm{~nm}$ for $d_{B}=2 \mathrm{~nm}$ in Fig. 6(a), whereas $L_{n} \equiv \pi\left(n-\frac{1}{2}\right) / k_{\text {coup }}$ (Sec. II C) predicts $L_{1}=$ $646 \mathrm{~nm}$ and $L_{2}=1939 \mathrm{~nm}$. Similarly, $L_{1}^{*}=1914 \mathrm{~nm}$ in Fig. 6(b) agrees with $L_{1}=1906 \mathrm{~nm}$ for $d_{B}=3 \mathrm{~nm}$.

Shown in Fig. 7 are the magnetic-field dependence of $G_{\uparrow, \downarrow}$ for $d_{B}=2$ and $3 \mathrm{~nm}$, where the values of $L$ are fixed at 646 and $1906 \mathrm{~nm}$, respectively. For $B>0$, we observe that $G_{\downarrow}$ becomes zero at $B=0.192$ and 0.177 T for $d_{B}=2$ and $3 \mathrm{~nm}$, respectively, which agree with the values of the anticrossing magnetic field $B_{a}$. For $B<0, G_{\uparrow}$ reaches zero at $B=-B_{a}$, while $G_{\downarrow}$ is kept close to unity, as is consistent with the symmetry consideration. In Fig. 7, we also recognize that the variation of $G_{\downarrow}\left(G_{\uparrow}\right)$ around $B=B_{a}$ $\left(B=-B_{a}\right)$ is more moderate for $d_{B}=2 \mathrm{~nm}$ than for $d_{B}=3 \mathrm{~nm}$. This is because the bonding and antibonding states $\left(1 / \sqrt{2}\left\{\left|\Psi_{\mathrm{QW} 1}\right\rangle \pm\left|\Psi_{\mathrm{QW} 2}\right\rangle\right\}\right)$ at $B= \pm B_{a}$ are more robust against the change of $B$ for $d_{B}=2 \mathrm{~nm}$ than for $d_{B}=3 \mathrm{~nm}$ because of the stronger $t_{\text {coup }}$. The range of the magnetic field $\Delta B$ around $B=B_{a}$ within which the bonding and antibonding states persist can be roughly estimated by equating $e \Delta B\langle z\rangle / \hbar$ to $k_{\text {coup. We obtain }}$ 

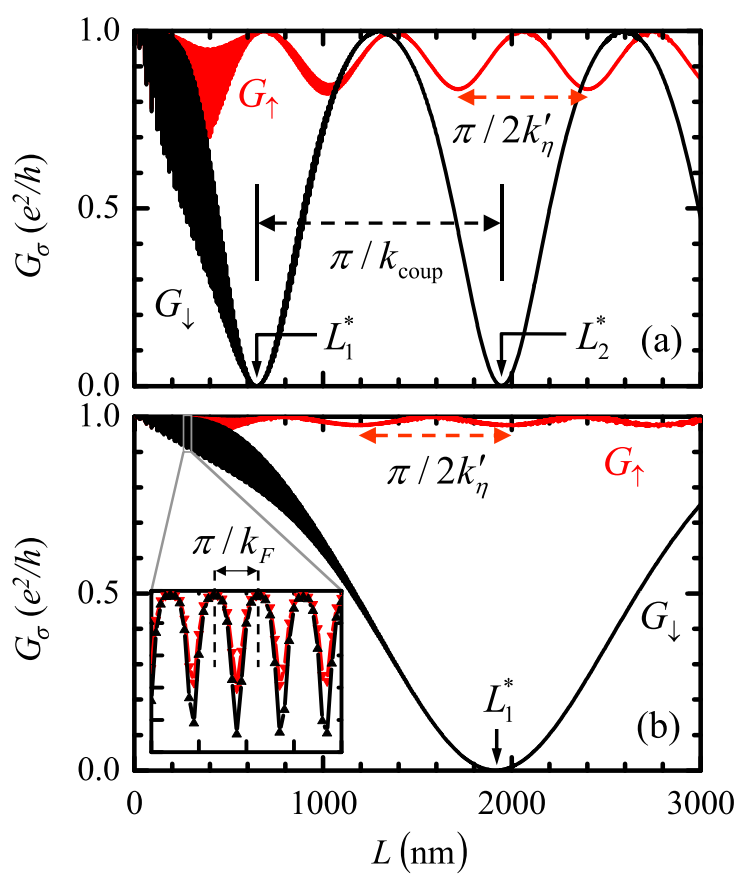

FIG. 6. Plots of the spin-dependent conductances $G_{\uparrow, \downarrow}$, calculated for two different barrier thicknesses (a) $d_{B}=2 \mathrm{~nm}$ and (b) $d_{B}=3 \mathrm{~nm}$ as a function of the device length $L$. The magnetic field is fixed at their anticrossing values $B_{a}=0.192 \mathrm{~T}$ for (a) and $0.177 \mathrm{~T}$ for (b). The inset in (b) is the magnified view of the main panel between 260 and $300 \mathrm{~nm}$ for the abscissa and between $0.9 e^{2} / h$ and $1.0 e^{2} / h$ for the ordinate, where the period of rapid oscillation is found to be $\pi / k_{F} . k_{\text {coup }}$ and $2 k_{\eta}^{\prime}$ are the $\Delta k$ values explained in Sec. III C, which are physically associated with the interwell coupling $t_{\text {coup }}$ and the interband Rashba spin splitting, respectively. $L_{n}^{*}(n=1$ or 2$)$ are the values of $L$ where the values of $G_{\downarrow}$ are at local minima.

$\Delta B=0.24$ and $0.075 \mathrm{~T}$ for $d_{B}=2 \mathrm{~nm}$ and $d_{B}=3 \mathrm{~nm}$, respectively, which agree well with the widths of conductance dips observed in Fig. 7.

In Figs. 6 and 7, there are features that cannot be explained by the conceptual argument in Sec. II C. The weak modulation of the spin-up conductance $G_{\uparrow}$ with period $\pi / 2 k_{\eta}^{\prime}\left(k_{\eta}^{\prime}=\sqrt{k_{\eta}^{2}+\frac{1}{4} k_{\text {coup }}^{2}}\right)$ in Fig. 6 is explained by the property of $T_{11}$ in the MR model (Sec. III C) setting $\Delta k=2 k_{\eta}^{\prime}$. The rapid oscillations in the spin-dependent conductance [inset in Fig. 6(b)] (i) are more pronounced for shorter $L$, (ii) disappear as $G_{\uparrow, \downarrow}$ approaches to zero or to the maximum value $e^{2} / h$, and (iii) damp away with increasing $L$. The period of these $(\Delta L \simeq 9.34 \mathrm{~nm})$, virtually independent of the value of $L$, is in good agreement with $\pi / k_{F}$, inferring some relation with the resonance phenomena associated with the multiple reflections of the electron between $x=0$ and $x=L$. We find that such reflections are actually caused by the finite probability amplitude of the electron wave function in $\mathrm{QW} 2$ (denoted as $A_{\mathrm{QW} 2}$ ) at $x=0$ and $L$. As $L$ approaches to $L_{n}^{*}$ or to $L_{n}^{*}+\pi / 2 k_{\text {coup }}$, where
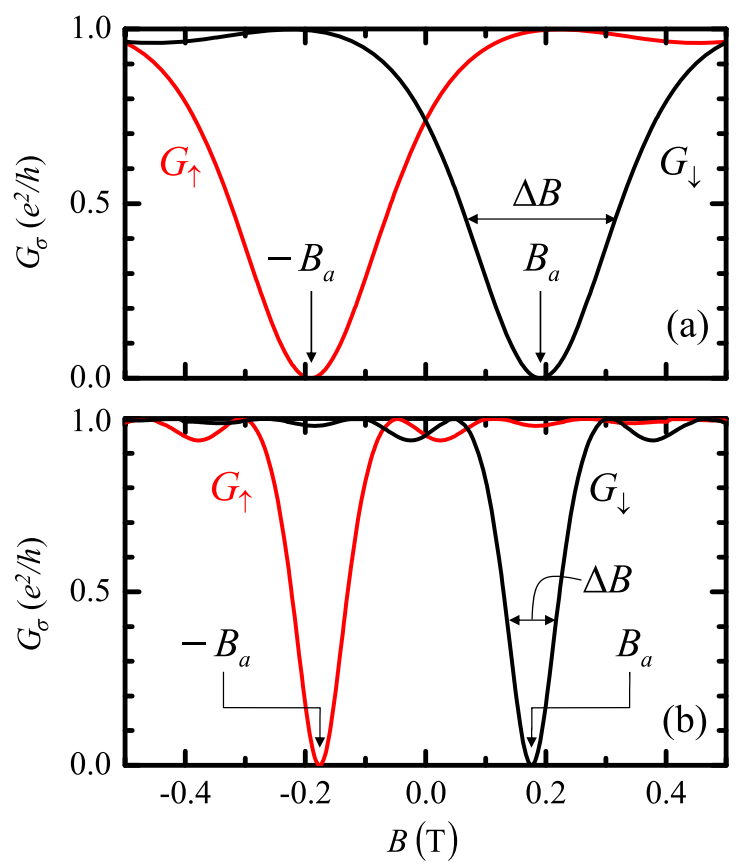

FIG. 7. The spin-dependent conductances as a function of the in-plane magnetic field $B$ for (a) $d_{B}=2 \mathrm{~nm}$ and (b) $d_{B}=3 \mathrm{~nm}$. The device lengths for $d_{B}=2$ and $3 \mathrm{~nm}$ are $L=646$ and $1906 \mathrm{~nm}$, respectively. The anticrossing magnetic fields $B_{a}$ for $d_{B}=2$ and $3 \mathrm{~nm}$ are 0.192 and $0.177 \mathrm{~T}$, respectively (indicated in the figures). See Sec. IV for the physical meaning of $\Delta B$.

$G_{\downarrow}$ reaches zero or $e^{2} / h$, respectively, $A_{\mathrm{QW} 2}$ vanishes at $x=0$ or $L$, hence the suppression of the reflections (the disappearance of the rapid oscillations). Damping of the rapid oscillations with increasing $L$ is caused by the finite width (denoted as $\delta k$ ) in the distribution of $k$ due to a nonzero temperature. This rapid oscillation persists if $\delta k L \ll 1$. Using $\delta k=k_{B} T(\partial E / \partial k)^{-1} \approx 8 \times 10^{-5} \mathrm{~m}^{-1}$ ( $T=5 \mathrm{~K}$ ), $L \ll 1.25 \mu \mathrm{m}$. This explains the decay of the rapid oscillation with increasing $L$.

The effect of the summation over $k_{y}$ in Eq. (3) is discussed next. In Fig. 8, we plot the spin polarization $P_{\text {total }}$ [as defined by Eq. (4)] as a function of $L$ for $d_{B}=$ $2 \mathrm{~nm}$ (a) and $3 \mathrm{~nm}$ (b), with and without the summation over $k_{y}$, where $B$ is fixed at 0.192 and $0.177 \mathrm{~T}$ for (a) and (b), respectively. We find that the spin-polarization values are reduced approximately to 0.4 even at $L$ 's close to $L_{n}$ 's. Thus, the summation over $k_{y}$ is indeed detrimental to the spin polarization. However, this effect is not strong enough to kill the net spin polarization completely. According to Ref. [5], the available experimental values for the bulk spin polarization in nonmagnetic semiconductors are about $\rho_{\mathrm{el}} / E_{x} \sim 1 \mu \mathrm{m}^{-3} / \mathrm{kV} \mathrm{m}^{-1}$, where the spin density $\rho_{\mathrm{el}}$ is divided by the exciting electric field $E_{x}$. The corresponding value in our device (in its leads) is conservatively estimated to be $\gtrsim 10^{3} \mu \mathrm{m}^{-3} / \mathrm{kV} \mathrm{m}^{-1}$ using $P_{\text {total }}=0.01$ (note $\left.\rho_{\text {el }} \propto P\right)$. This potentially offers a $>10^{4}$ enhancement in 

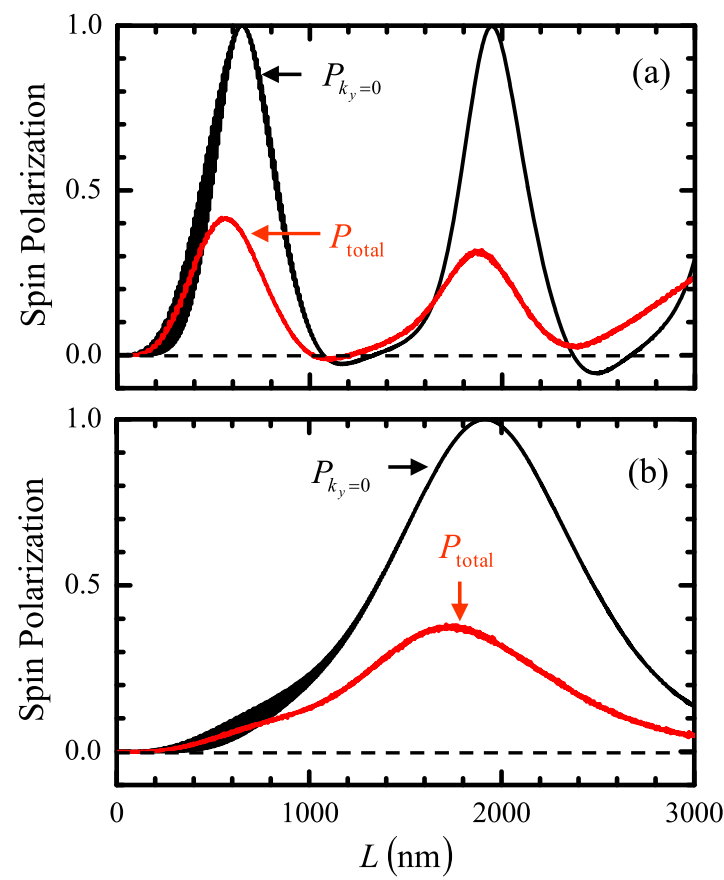

FIG. 8. Plots of the spin polarization $P_{\text {total }}$ [Eq. (4)], after summing up over the allowed $k_{y}$ 's in Eq. (2), as a function of $L$ for (a) $d_{B}=2 \mathrm{~nm}$ and (b) $d_{B}=3 \mathrm{~nm}$. The magnetic fields are fixed at $0.192 \mathrm{~T}$ for (a) and $0.177 \mathrm{~T}$ for (b) ( $B_{a}$ values). Results with $k_{y}=0$, calculated using $G_{\uparrow, \downarrow}$ in Fig. 6, are also plotted for comparison (denoted by $P_{k_{y}=0}$ ).

the bulk spin polarization relative to the existing experimental values. We also finally note our prospect of obtaining a net spin polarization purely electrically in our multichannelized device [42], which defines the direction of our future research.

\section{CONCLUSION}

We propose a lateral spin-blockade device using a $\mathrm{In}_{0.53} \mathrm{Ga}_{0.47} \mathrm{As} / \mathrm{In}_{0.52} \mathrm{Al}_{0.48} \mathrm{As} \mathrm{DQW}$, where the interband Rashba effect becomes important. The principle of the spinblocking effect in the proposed device is the spin-selective matching of the Fermi circle edges in $\mathbf{k}$ space between the two QWs, which is made possible by the in-plane magnetic field $\mathbf{B}=\left(0, B_{a}, 0\right)$. The superposition of the bonding and antibonding wave functions for the selected spin (e.g., spin down) results in precessional motions of electrons between the QW1 and QW2. The electrons in such motions can be blocked by the etched walls made in QW2.

We reiterate the features of the proposed spin device. (i) It enhances the electromagnetically controlled RashbaEdelstein effect $[3,4]$, generating a bulk spin polarization at either lead of the device depending on the direction of the current. (ii) Well-established semiconductor technologies are readily applicable for both the design (property prediction) and fabrication of the proposed device. (iii) Some deterioration in the net spin polarization is found when including the finite $k_{y}$ components. Despite this last point, the bulk spin polarization generated in the proposed device is fairly large, i.e., approximately $10^{4}$ enhancement relative to the available experimental values, which opens the way to the future spintronics applications widely.

\section{ACKNOWLEDGMENTS}

The authors thank Dr. J. C. Egues for valuable input and advice in revising the manuscript. This work was supported by KAKENHI, Grant-in-Aid for Scientific Research (B), No. 23360001.

[1] S. Datta and B. Das, Electronic analog of the electro-optic modulator, Appl. Phys. Lett. 56, 665 (1990).

[2] S. K. Upadhyay, R. N. Louie, and R. A. Buhrman, Spin filtering by ultrathin ferromagnetic films, Appl. Phys. Lett. 74, 3881 (1999).

[3] V. M. Edelstein, Spin polarization of conduction electrons induced by electric current in two-dimensional asymmetric electron systems, Solid State Commun. 73, 233 (1990).

[4] A. G. Aronov and Y. B. Lyanda-Geller, Nuclear electric resonance and orientation of carrier spins by an electric field, JETP Lett. 50, 431 (1989).

[5] Y. K. Kato, R. C. Myers, A. C. Gossard, and D. D. Awschalom, Current-Induced Spin Polarization in Strained Semiconductors, Phys. Rev. Lett. 93, 176601 (2004).

[6] A. Yu. Silov, P. A. Blajnov, J. H. Wolter, R. Hey, K. H. Ploog, and N.S. Averkiev, Current-induced spin polarization at a single heterojunction, Appl. Phys. Lett. 85, 5929 (2004).

[7] K. Shen, G. Vignale, and R. Raimondi, Microscopic Theory of the Inverse Edelstein Effect, Phys. Rev. Lett. 112, 096601 (2014).

[8] J. E. Hirsch, Spin Hall Effect, Phys. Rev. Lett. 83, 1834 (1999).

[9] S. Murakami, N. Nagaosa, and S.-C. Zhang, Dissipationless Quantum spin current at room temperature, Science 301, 1348 (2003).

[10] Y. K. Kato, R. C. Myers, A. C. Gossard, and D. D. Awschalom, Observation of the spin Hall effect in semiconductors, Science 306, 1910 (2004).

[11] J. Wunderlich, B. Kaestner, J. Sinova, and T. Jungwirth, Experimental Observation of the Spin-Hall Effect in a TwoDimensional Spin-Orbit Coupled Semiconductor System, Phys. Rev. Lett. 94, 047204 (2005).

[12] A. Voskoboynikov, S. S. Lin, C. P. Lee, and O. Tretyak, Spin-polarized electronic current in resonant tunneling heterostructures, J. Appl. Phys. 87, 387 (2000).

[13] D. Z.-Y. Ting and X. Cartoixà, Resonant interband tunneling spin filter, Appl. Phys. Lett. 81, 4198 (2002).

[14] T. Koga, J. Nitta, H. Takayanagi, and S. Datta, Spin-Filter Device Based on the Rashba Effect Using a Nonmagnetic Resonant Tunneling Diode, Phys. Rev. Lett. 88, 126601 (2002). 
[15] M. Eto, T. Hayashi, and Y. Kurotani, Spin polarization at semiconductor point contacts in absence of magnetic field, J. Phys. Soc. Jpn. 74, 1934 (2005).

[16] P. Debray, S. M. S. Rahman, J. Wan, R. S. Newrock, M. Cahay, A. T. Ngo, S. E. Ulloa, S. T. Herbert, M. Muhammad, and M. Johnson, All-electric quantum point contact spinpolarizer, Nat. Nanotechnol. 4, 759 (2009).

[17] M. Kohda, S. Nakamura, Y. Nishihara, K. Kobayashi, T. Ono, J. Ohe, Y. Tokura, T. Mineno, and J. Nitta, Spin-orbit induced electronic spin separation in semiconductor nanostructures, Nat. Commun. 3, 1082 (2012).

[18] S. Kim, Y. Hashimoto, Y. Iye, and S. Katsumoto, Evidence of spin-filtering in quantum constrictions with spin-orbit interaction, J. Phys. Soc. Jpn. 81, 054706 (2012).

[19] E. Bernardes, J. Schliemann, M. Lee, J. C. Egues, and D. Loss, Spin-Orbit Interaction in Symmetric Wells with Two Subbands, Phys. Rev. Lett. 99, 076603 (2007).

[20] R. S. Calsaverini, E. Bernardes, J. C. Egues, and D. Loss, Intersubband-induced spin-orbit interaction in quantum wells, Phys. Rev. B 78, 155313 (2008).

[21] J. Fu and J. C. Egues, Spin-orbit interaction in GaAs wells: From one to two subbands, Phys. Rev. B 91, 075408 (2015).

[22] U. Ekenberg and D. M. Gvozdić, Analysis of electric-fieldinduced spin splitting in wide modulation-doped quantum wells, Phys. Rev. B 78, 205317 (2008).

[23] T. Matsuura, S. Faniel, N. Monta, and T. Koga, Magnetic control of Rashba splittings in symmetric InAs quantum wells, Physica E (Amsterdam) 42, 2707 (2010).

[24] T. Koga, T. Matsuura, S. Faniel, S. Souma, S. Mineshige, Y. Sekine, and H. Sugiyama, Beating analysis of Shubnikov de Haas oscillation in $\operatorname{In}_{0.53} \mathrm{Ga}_{0.47}$ As double quantum well toward spin filter applications, IEICE Trans. Electron. E95.C, 770 (2012).

[25] J. Nitta, T. Akazaki, H. Takayanagi, and T. Enoki, Gate Control of Spin-Orbit Interaction in an Inverted $\mathrm{In}_{0.53} \mathrm{Ga}_{0.47} \mathrm{As} / \mathrm{In}_{0.52} \mathrm{Al}_{0.48}$ As Heterostructure, Phys. Rev. Lett. 78, 1335 (1997).

[26] T. Koga, J. Nitta, T. Akazaki, and H. Takayanagi, Rashba Spin-Orbit Coupling Probed by the Weak Antilocalization Analysis in InAlAs/InGaAs/InAlAs Quantum Wells as a Function of Quantum Well Asymmetry, Phys. Rev. Lett. 89, 046801 (2002).

[27] S. Faniel, T. Matsuura, S. Mineshige, Y. Sekine, and T. Koga, Determination of spin-orbit coefficients in semiconductor quantum wells, Phys. Rev. B 83, 115309 (2011).

[28] S. Nakosai, Y. Tanaka, and N. Nagaosa, Topological Superconductivity in Bilayer Rashba System, Phys. Rev. Lett. 108, 147003 (2012).

[29] S. I. Erlingsson and J. C. Egues, All-electron topological insulator in InAs double wells, Phys. Rev. B 91, 035312 (2015).
[30] L. Esaki, Do-it-yourself quantum mechanics in lowdimensional structures, Phys. Scr. T42, 102 (1992).

[31] H. Kroemer, Band offsets and chemical bonding: the basis for heterostructure applications, Phys. Scr. T68, 10 (1996).

[32] T. Koga, J. Nitta, T. Akazaki, and H. Takayanagi, Conditions for the spin rectification phenomena predicted for semiconducting triple barrier structures in the presence of the Rashba spin-orbit coupling, Jpn. J. Appl. Phys. 41, 2501 (2002).

[33] We obtain $\left(e^{2} B^{2} z^{2} / 2 m_{\|}^{*}\right)=3.67 \mu \mathrm{eV}, \eta k_{x}=1.06 \mathrm{meV}$, $\eta e B z / \hbar=6.68 \mu \mathrm{eV}$, and $-g^{*} \mu_{B} B / 2=17.4 \mu \mathrm{eV}$ using $m_{\|}^{*} / m_{e}=0.047, g^{*}=-3, B=0.2 \mathrm{~T}, \eta=3.14 \times 10^{-12} \mathrm{eVm}$, $k_{x}=3.36 \times 10^{8} \mathrm{~m}^{-1}$, and $z=7 \mathrm{~nm}$. See J. Nitta, Y. Lin, T. Akazaki, and T. Koga, Gate-controlled electron $g$ factor in an InAs-inserted-channel $\operatorname{In}_{0.53} \mathrm{Ga}_{0.47} \mathrm{As} / \mathrm{In}_{0.52} \mathrm{Al}_{0.48}$ As heterostructure, Appl. Phys. Lett. 83, 4565 (2003); F. E. Meijer, A. F. Morpurgo, T. M. Klapwijk, T. Koga, and J. Nitta, Competition between spin-orbit interaction and Zeeman coupling in Rashba two-dimensional electron gases, Phys. Rev. B 70, 201307 (2004) for $g^{*}$ values in (In, Ga)As/(In, Al)As QWs.

[34] R. Winkler, Spin-Orbit Coupling Effects in Two-Dimensional Electron and Hole Systems (Springer, Berlin, 2003).

[35] G. Engels, J. Lange, Th. Schäpers, and H. Lüth, Experimental and theoretical approach to spin splitting in modulationdoped $\mathrm{In}_{x} \mathrm{Ga}_{1-x}$ As/InP quantum wells for $B \rightarrow 0$, Phys. Rev. B 55, R1958 (1997).

[36] A. Zaslavsky, Y. P. Li, D. C. Tsui, M. Santos, and M. Shayegan, Transport in transverse magnetic fields in resonant tunneling structures, Phys. Rev. B 42, 1374 (1990).

[37] C.-M. Hu and D. Heitmann, Bilayer quantum transistor, Appl. Phys. Lett. 77, 1475 (2000).

[38] F. Zhai and H.Q. Xu, Symmetry of Spin Transport in Two-Terminal Waveguides with a Spin-Orbital Interaction and Magnetic Field Modulations, Phys. Rev. Lett. 94, 246601 (2005).

[39] M. Büttiker, Y. Imry, R. Landauer, and S. Pinhas, Generalized many-channel conductance formula with application to small rings, Phys. Rev. B 31, 6207 (1985).

[40] B. K. Nikolić, P. Zarbo, and S. Souma, in The Oxford Handbook on Nanoscience and Technology, edited by A. V. Narlikar and Y. Y. Fu (Oxford University, New York, 2010), Vol. I, Chap. 24, p. 814.

[41] $m_{z}^{*} \operatorname{In}_{0.53} \mathrm{Ga}_{0.47} \mathrm{As}=0.047 m_{e}$ is used in this study. The dependences of $t_{\text {coup }}$ and $\langle z\rangle$ on $d_{B}$ are given by the following empirical formulas: $t_{\text {coup }}(\mathrm{eV})=11.66 e^{-1.09 d_{B}}$ and $\langle z\rangle(\mathrm{nm})=$ $-1.105 e^{-1.12 d_{B}}+5.744+d_{B} / 2$, respectively, where the unit for $d_{B}$ is nanometer.

[42] $P>11 \%$ is obtained in our preliminary work: A. Sawada and T. Koga (unpublished). 